\title{
Tuberculosis: History, Epidemiology, Antitubercular Drugs and Plant-based Alternatives
}

\author{
B. TIWARI* ${ }^{*}$ SUNITA SHAILAJAN ${ }^{1}$, S. MENON² AND SAVITA KULKARNI ${ }^{3}$
}

Enaltec Labs Pvt. Ltd., Analytical Research and Development Department, Plot No. W-59A, Anand Nagar, Additional MIDC, Ambernath-421 506, ${ }^{1}$ Herbal Research Laboratory, Ramnarain Ruia College, Matunga-400 019, ${ }^{2}$ Institute for Advanced Training and Research in Interdisciplinary Sciences, TDM Laboratory, Sion (East)-400 022, ${ }^{3}$ Radiation Medicine Centre, BioMedical Group, Bhabha Atomic Research Centre, Trombay-400 085, Mumbai, India

\section{Tiwari, et al.: A Brief Review on Tuberculosis}

\begin{abstract}
The article is a brief review on certain important aspects of tuberculosis. A good number of medicinal plants and their chemical constituents are reported to have antimycobacterial activity comparable to the existing antitubercular drugs or sometimes even better in efficacy. The present review covers the literature published concerning medicinal plants and plant-based active constituents showing both immunomodulatory and antimycobacterial activity. These plants might eventually be studied and screened with a well-defined strategy to develop effective new drugs against tuberculosis.
\end{abstract}

Key words: Tuberculosis, chemotherapy, immunotherapy, alternative therapy

Millions of people of all age groups die each year worldwide from diseases such as chest and respiratory disorders, bacteraemia, wound suppuration, diarrhoea, dysentery and tuberculosis (TB). Additionally, millions suffer from several long-standing chronic infections such as $\mathrm{TB}^{[1]}$. TB is characterized as a chronic bacterial infection caused by Mycobacterium tuberculosis, an aerobic acid-fast bacillus. $M$. tuberculosis, the causative agent of human TB, has an exclusive tropism for this host ${ }^{[2]}$ and is the most notable member amongst the five different species of genus Mycobacterium, M. tuberculosis, M. canettii, $M$. africanum, M. microti and $M$. bovis. In contrast, $M$. bovis, the etiologic agent of bovine TB, causes only 5-10\% of all human TB cases $^{[3,4]}$ while $M$. africanum is responsible for half of the TB cases in West Africa ${ }^{[5]}$. TB is one of the most devastating bacterial disease with high rates of morbidity and mortality ${ }^{[6]}$ and it continued to be a major health concern all over the world ${ }^{[7]}$ ranking as the second leading cause of death from an infectious disease worldwide, after the human immunodeficiency virus (HIV $)^{[8]}$. TB is the leading killer of HIV-positive people causing one-fourth of all HIV-related deaths. It typically affects the lungs (pulmonary TB) but can affect other sites as well (extra-pulmonary TB). It spreads through the air when people infected with pulmonary TB expel bacteria by coughing ${ }^{[8]}$. Because of the worldwide health problem that TB represents, it is essential to find new drugs that allow better control of TB. In the present review, various aspects of TB were covered along with pharmacological information on 11 medicinal plants and 11 phytochemical constituents with potential immunomodulatory and antimycobacterial activities. These medicinal plants and phytochemical constituents appear to be promising leads for further investigations that would help development of newer antitubercular (antiTB) drugs.

\section{Historical background:}

TB is one of the oldest infectious diseases affecting mankind ${ }^{[8]}$. TB is a disease of antiquity, which is thought to have evolved sometime between the seventh and sixth millennia $\mathrm{BC}^{[9-12]}$. The earliest references to TB can be found in the ancient Indian scriptures Vedas, where it was referred to as Yakshma (wasting disease). Description of a TB-like disease has been documented in ancient Chinese and Arabic literature also ${ }^{[8]}$. Robert Koch announced the discovery of the tubercle bacillus during the monthly evening meeting

This is an open access article distributed under the terms of the Creative Commons Attribution-NonCommercial-ShareAlike 3.0 License, which allows others to remix, tweak, and build upon the work non-commercially, as long as the author is credited and the new creations are licensed under the identical terms

Accepted 20 January 2019

Revised 21 May 2018

Received 06 June 2017

*Address for correspondence

E-mail: bhaveshtiwari23@gmail.com

March-April 2019 
of the Berlin Physiological Society on March 24, 1882. Commemorating the centenary of this event, since 1982, March 24 is being celebrated as "World TB day ${ }^{[8]}$.

\section{Epidemiology, global burden and Indian scenario:}

The global burden of TB was reported in a report published by World Health Organization (WHO) in $2016^{[13]}$. In 2015, about $60 \%$ of reported TB cases occurred in 6 countries namely India, Indonesia, China, Nigeria, Pakistan and South Africa. The list of countries with 3 TB high-burden cases, TB, multidrug-resistant TB (MDR-TB), TB/HIV has also been published by WHO in global TB report. An estimated $11 \%$ of the incident TB cases were HIV-positive. In addition to the 1.4 million TB deaths among HIV-negative, there were 0.4 million deaths from TB among HIV-positive. In 2015, there were an estimated 10.4 million TB cases worldwide, of which $56 \%$ were among men, $34 \%$ women and $10 \%$ children. There were an estimated 0.48 million new cases of MDR-TB. India, China and the Russian Federation accounted for $45 \%$ of the combined total of MDR-TB cases. On average, an estimated $9.5 \%$ of patients with MDR-TB had extensively drug-resistant TB.

India is the second-most populous country in the world. It accounts for more than one quarter of the global incident TB cases and deaths annually ${ }^{[14]}$. Globally, in each year India has the highest number of new TB cases, MDR-TB and deaths related to $\mathrm{TB}^{[15]}$. In 2014, the estimated incidence of TB cases in India was nearly 2.2 million while the estimated TB prevalence figure was 2.5 million. An estimated $5 \%$ of the incident TB cases were HIV-positive. In India, $2.2 \%$ of new TB cases were estimated to have had MDR-TB ${ }^{[16]}$.

\section{Infection:}

TB infection is a cascade mechanism, which initiates an intracellular signalling that culminates in a proinflammatory response (beneficial to the host) or dampens the innate immune response, which is beneficial to the pathogen ${ }^{[17]}$. The infection process of $\mathrm{TB}$ can be divided into 3 different interrelated stages ${ }^{[18]}$. The first stage is the aerosol transmission of the droplets containing $M$. tuberculosis from an infected individual to a healthy individual. In the primary infection, M. tuberculosis multiplies in the lungs and causes mild inflammation. Although alveolar macrophages are thought to be an effective barrier to contain pathogens, M. tuberculosis has evolved various mechanisms to evade and survive within these cells ${ }^{[19]}$. In the next stage of infection, M. tuberculosis bacilli that escape the cytolytic effects of the alveolar macrophages, multiply and result in their destruction. After 6-8 $\mathrm{w}$ of infection, antigen presenting dendritic cells travel to lymph nodes where activated T-lymphocytes migrate to the site of infection and proliferate to form an early stage granuloma. This marks the persistent stage of infection (latency), where the growth and spread of bacteria into additional tissue sites are limited ${ }^{[20]}$. The third and final stage is when latent and controlled M. tuberculosis infection is reactivated due to a decline in the host's immunity and failure to develop and maintain immune signals $^{[21]}$. Under these circumstances, the granuloma structure disrupts and results in the lung cavitation and pulmonary $\mathrm{TB}^{[22,23]}$. The spread of M. tuberculosis bacilli may rapidly result in extra-pulmonary $\mathrm{TB}^{[24]}$.

\section{Factors responsible for the emergence of TB:}

A number of factors make people more susceptible to TB infection. The risk factors for developing disease following infection with $M$. tuberculosis include factors that affect immunity ${ }^{[24]}$. Some of them are age and sex ${ }^{[25]}$, nutrition $^{[15]}$, drug-induced immunosuppression ${ }^{[26]}$ and poverty ${ }^{[27]}$.

\section{Symptoms and diagnosis:}

Cough, blood-stained sputum, loss of weight, fever/ sweating, pain in the chest are some important symptoms of TB. Other symptoms may include breathlessness, weakness, localized wheeze, frequent colds, tiredness, loss of appetite. These symptoms could be due to some other illness too. Hence, examination of sputum for the confirmation of TB infection is a must $^{[24]}$. Diagnosis of TB is mainly based on the clinical features, histopathology and demonstration of acid-fast bacilli from the clinical specimens ${ }^{[28]}$. TB diagnosis depends primarily on sputum smear microscopy, chest radiography and tuberculin skin tests ${ }^{[29]}$. Several rapid methods based on lipid analysis, specific gene probes, polymerase chain reaction-restriction fragment length polymorphism methods and ribosomal RNA sequencing have also been used for the diagnosis of $\mathrm{TB}^{[28]}$.

\section{AntiTB drugs and their adverse effects:}

The antiTB drugs are classified in five groups as mentioned in Table $1^{[30-32]}$. The targets of each member of the first-line drugs have been identified except for pyrazinamide, whose mechanism remains controversia $^{[33]}$. Directly-observed treatment; the most 
cost effective way to stop the spread of $\mathrm{TB}$, is one of the five key elements in the WHO global TB control programme-strategy ${ }^{[34]}$. The use of antiTB drugs is also associated with significant side-effects such as hepatitis, neuropathy, hypersensitivity syndrome, thrombocytopenia, fever, dyspepsia ${ }^{[35-37]}$. Such adverse effects are responsible for termination of therapy during the intensive phase, which makes the situation even more difficult to cure ${ }^{[38]}$. Bacterial resistance to one or more antiTB drugs has resulted in resistant forms of TB (Table 2). The emergence of such resistant forms of TB poses formidable challenges to global TB control efforts ${ }^{[39,40]}$. Thus, there is an urgent need of novel antiTB drugs, which are safe, able to shorten the course of treatment, effective against drug-resistant strains and latent TB infection.

\section{Role of immunomodulators in TB control:}

In view of the limited protection against $\mathrm{TB}$ afforded by Bacillus Calmette-Guérin vaccination, attempts are being made to develop some more effective alternative ${ }^{[41,42]}$. Immunotherapy for $\mathrm{TB}$ enhances the treatment success of resistant forms of TB, shortens the treatment course for chemo-sensitive TB, reduces TB recurrence after chemotherapy by improving immunity and alleviates immune pathological damage ${ }^{[43-45]}$. The immunotherapy strategy for $\mathrm{TB}$ includes restoring the balance of $\mathrm{T}$ helper cells (enhancing Th1 and suppressing Th2 response) by altering the dominant bacteriostatic response to a bactericidal response ${ }^{[46,47]}$. The use of immunotherapy for TB lacks efficacy in clinical trials and also associated with severe side effects such as hematopoietic toxicity, liver damage and muscle necrosis, teratogenicity, peripheral neuropathy, hypertension, hyperglycaemia ${ }^{[48-51]}$. Therefore, exploring the vast untapped natural resource for TB control as a safer alternative appears a valid option, which can reduce the possibility of generation of drug resistant bacteria, reduce the treatment length, possibility of re-infection and reactivation ${ }^{[52,53]}$.

\section{Plants as immunomodulatory and antimycobacterial agents:}

In every region, based on the climatic and geographic conditions, special medicinal plants grow and many of them have unique medicinal properties ${ }^{[54]}$. Due to the adverse effects of modern drugs and therapies, plants have been a common resource of medicaments in the treatment of a wide range of ailments ${ }^{[55,56]}$. Therefore, a number of pharmaceutical products derived from plants serve as cheap and safe alternative ${ }^{[57]}$.

Failure to control or resolve infectious disease such as TB often results from an inappropriate rather than insufficient immune response ${ }^{[58-60]}$. In this regard, the immune system can be stimulated by natural products of plant origin. A number of medicinal plants used in traditional medicine ${ }^{[60,61]}$ and their several active substances have also been reported to modulate the

TABLE 1: DIFFERENT GROUPS OF ANTITUBERCULAR DRUGS WITH SUITABLE EXAMPLES

\begin{tabular}{|c|c|c|}
\hline Description & Group & Examples \\
\hline \multirow[t]{2}{*}{ First-line } & 1 & Oral drugs (isoniazid, pyrazinamide, ethambutol, rifampicin, rifabutin) \\
\hline & 2 & $\begin{array}{l}\text { Injectable amino-glycosides (kanamycin, amikacin, streptomycin); injectable polypeptides } \\
\text { (capreomycin, viomycin) }\end{array}$ \\
\hline \multirow[t]{2}{*}{ Second-line } & 3 & $\begin{array}{c}\text { Oral and injectable fluoroquinolones (ciprofloxacin, levofloxacin, moxifloxacin, ofloxacin, } \\
\text { gatifloxacin) }\end{array}$ \\
\hline & 4 & Oral drugs (para-aminosalicylic acid, cycloserine, terizidone, ethionamide, prothionamide) \\
\hline Third-line & 5 & $\begin{array}{l}\text { Drugs with unclear efficacy or undefined role (clofazimine, linezolid, amoxicillin plus clavulanate, } \\
\text { imipenem plus cilastatin, TMC 207, nitroimidazoles) }\end{array}$ \\
\hline
\end{tabular}

TABLE 2: DRUG RESISTANT TUBERCULOSIS AND ASSOCIATED EFFECTS

\begin{tabular}{|c|c|c|}
\hline Resistant forms of TB & $\begin{array}{l}\text { Resistance to } \\
\text { antiTB drugs }\end{array}$ & $\begin{array}{l}\text { Effect (treatment is expensive and toxic for each resistant form } \\
\text { of TB) }\end{array}$ \\
\hline Multidrug-resistant TB & Isoniazid and rifampicin & Worse cure rate $(40-80 \%)$ \\
\hline $\begin{array}{l}\text { Extensively drug-resistant } \\
\text { TB }\end{array}$ & Group 2 and 3 drugs & Cure and survival rates are worse than multidrug-resistant TB \\
\hline $\begin{array}{l}\text { Beyond extensively } \\
\text { drug-resistant TB }\end{array}$ & $\begin{array}{c}\text { Group } 1,2,3 \text { and } 4 \\
\text { drugs }\end{array}$ & $\begin{array}{l}\text { Cure and survival rates could be worse than extensively drug- } \\
\text { resistant TB }\end{array}$ \\
\hline Extremely drug-resistant TB & Group 1-5 drugs & $\begin{array}{c}\text { Cure and survival rates could be worse than beyond extensively } \\
\text { drug-resistant TB }\end{array}$ \\
\hline Totally drug-resistant TB & Group 1-5 drugs & $\begin{array}{c}\text { Cure and survival rates could be worse than extremely drug- } \\
\text { resistant TB }\end{array}$ \\
\hline
\end{tabular}


immune response ${ }^{[62-66]}$. It has been discovered that the plant extracts possess antimycobacterial activity. The crude $80 \%$ methanol extracts of Ocimum basilicum seeds, Calpurnia aurea roots, and leaves of Croton macrostachyus, Artemisia abyssinica and Eucalyptus camaldulensis showed promising antimycobacterial activity against $M$. tuberculosi ${ }^{[67]}$. Ethiopian plants like Allium ursinum bulb, Dodonaea anguistifolia leaves and Pterolobium stellatum leaves have been traditionally used to treat TB and related symptoms in Northern part of Ethiopia. Their traditional claim was experimentally validated using $80 \%$ ethanol extracts against $M$. tuberculosis ${ }^{[68]}$. Plants are also a promising source of antimycobacterial compounds, which may have important role in the chemotherapy of $\mathrm{TB}$ and other respiratory tract infections. Various major groups of phytochemical constituents like alkaloids, flavonoids, chalcones, coumarins, lignans, phenols, terpenes, chromones, alkanes and alkenes found in plant extracts have been reported to possess antimycobacterial activity. It has been reported that the most effective isolated compounds from plants are plumbagin, maritinone, aloe-emodin, epigallocatechin and umckalin, tiliacorinine, mauritine, globiferin, beilschmin, 7-methyljuglone ${ }^{[69,70]}$. The traditional knowledge of plants is becoming an important asset in developing newer and better drugg ${ }^{[71]}$. Recently, the antimycobacterial activity of a plant extracts rich in allicin and ajoene has been reported ${ }^{[72]}$. A considerable number of plant species, which have been mentioned in Ayurveda for the treatment of TB and related disorders were found more active with minimum inhibitory concentration (MIC) value between $10-100 \mu \mathrm{g} / \mathrm{ml}^{[73]}$. Literature also reported the antimycobacterial activity of many classes of natural products ${ }^{[0,74-77]}$.

In this review, pharmacological information on 11 medicinal plants and 11 phytochemical constituents was compiled (fig. 1) with reference to the immunomodulatory (in vitro in preclinical models/in vivo in cell lines) and antimycobacterial (in vitro against drug-susceptible strain $M$. tuberculosis H37Rv) activities. These plants were from a wide range of families such as Rutaceae, Acanthaceae, Amaryllidaceae, Apiaceae, Clusiaceae, Lamiaceae,

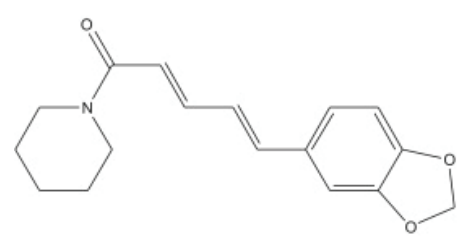

A

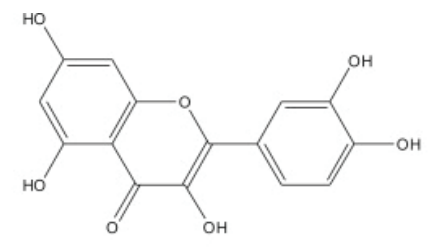

B

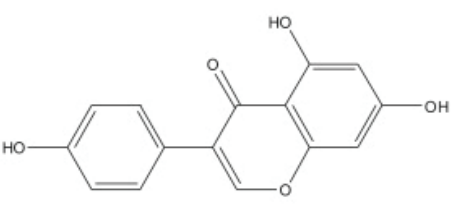

C

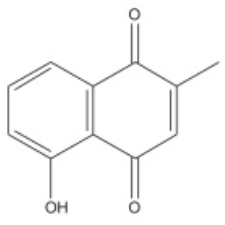

D

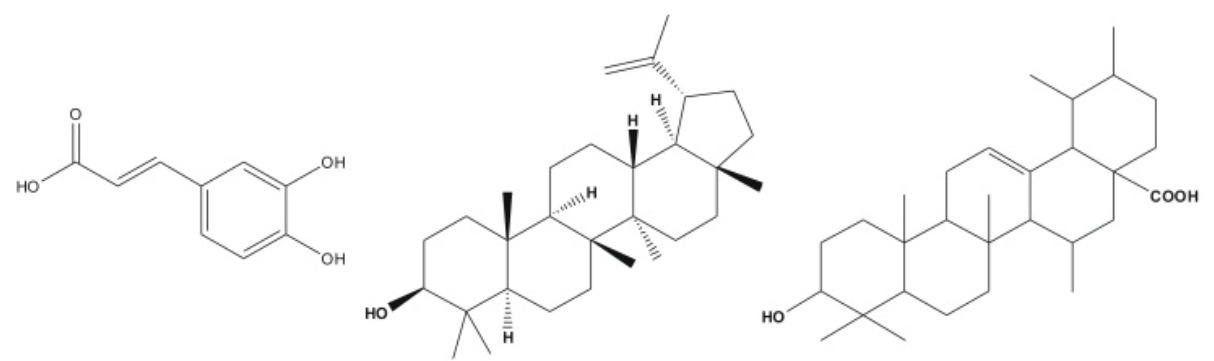

$\mathbf{E}$

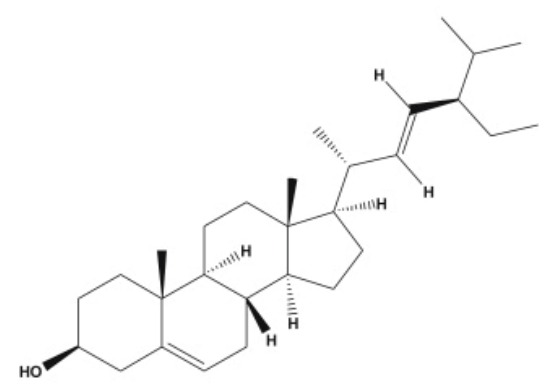

I
F

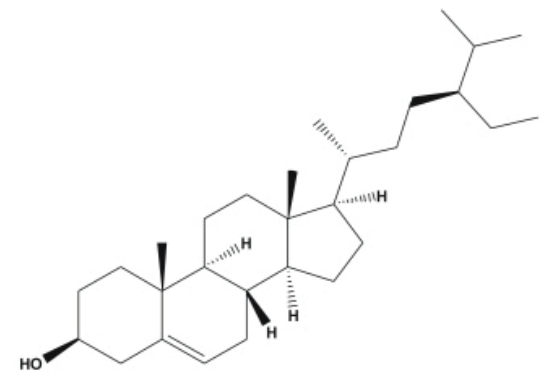

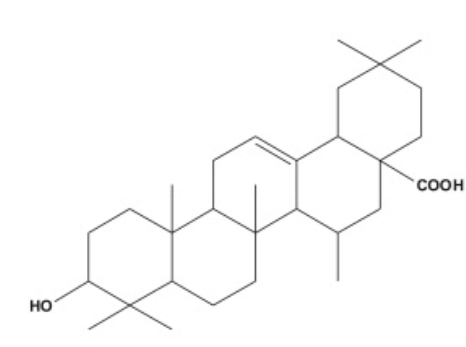

H

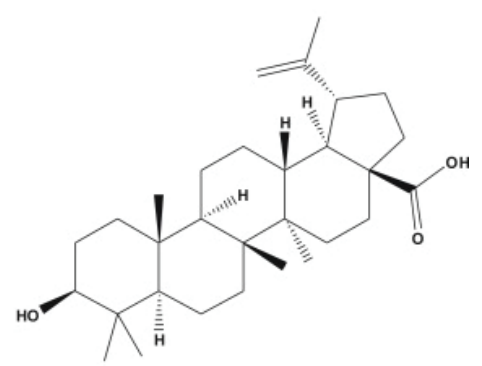

$\mathbf{K}$

Fig. 1: Phytochemical constituents having immunomodulatory and antimycobacterial potential

A: piperine, B: quercetin, C: genistein, D: plumbagin, E: caffeic acid, F: lupeol, G: ursolic acid; H: oleanolic acid, I: stigmasterol, J: beta-sitosterol, K: betulinic acid 
Leguminosae, Verbenaceae and Solanaceae. The reported pharmacological activity of these plants has been summarized in Table 3 and 4, which is further discussed hereafter.

Administration of the methanol extract of Aegle marmelos fruits significantly stimulated the immune system by acting through cellular and humoral immunity in experimental animals at a dose of $100 \mathrm{mg} / \mathrm{kg}^{[78]}$. In an another study, hexane and acetone extract of Aegle marmelos leaves showed MIC value of $50 \mu \mathrm{g} / \mathrm{ml}$, whereas methanol extract showed MIC value of $100 \mu \mathrm{g} / \mathrm{ml}$ against $\mathrm{H} 37 \mathrm{Rv}$ strain of M. tuberculosis by Alamar blue assay and thus indicating their antimycobacterial activity ${ }^{[79]}$. An immunomodulatory study on the aqueous extract of A. sativum bulbs showed significant increase in total leukocytes count at $100 \mathrm{mg} / \mathrm{kg}$ in Wistar albino rats ${ }^{[80]}$. In addition, its aqueous extract also showed promising antimycobacterial activity when evaluated against M. tuberculosis H37Rv strain using REMA and was more significant compared to the standard drugs ${ }^{[72]}$. Puri et al. reported that the administration of the ethanol extract of Andrographis paniculata whole plant induced significant stimulation of the antibodies and delayed type hypersensitivity response to sheep red blood cells stimulated $\mathrm{Balb} / \mathrm{c}$ mice. It also stimulated antigen specific as well as non-specific immune response suggesting its immunostimulatory

TABLE 3: MEDICINAL PLANTS AND EXTRACTS WITH IMMUNOMODULATORY AND ANTIMYCOBACTERIAL ACTIVITIES

\begin{tabular}{|c|c|c|c|c|}
\hline \multirow{2}{*}{$\begin{array}{l}\text { Plant name } \\
\text { (family) }\end{array}$} & \multicolumn{2}{|c|}{ Immunomodulatory activity } & \multicolumn{2}{|c|}{ Antimycobacterial activity } \\
\hline & $\begin{array}{c}\text { Extract } \\
\text { (plant part) }\end{array}$ & Results & $\begin{array}{c}\text { Extract } \\
\text { (plant part) }\end{array}$ & Results \\
\hline $\begin{array}{l}\text { Aegle marmelos (L.) } \\
\text { Correa (Rutaceae) }\end{array}$ & $\begin{array}{l}\text { Methanol } \\
\text { (fruits) }\end{array}$ & $\begin{array}{l}\text { Significant increase in biochemical } \\
\text { parameters at } 500 \mathrm{mg} / \mathrm{kg}, \mathrm{p} . \mathrm{o} ., \text { in } \\
\text { Wistar albino rats } \\
\text { [8] }\end{array}$ & $\begin{array}{l}\text { Hexane, acetone and } \\
\text { methanol (leaves) }\end{array}$ & $\begin{array}{c}\text { MIC } 100 \mu \mathrm{g} / \mathrm{ml} \text { using } \\
\text { MABA }^{[79]}\end{array}$ \\
\hline $\begin{array}{l}\text { Allium sativum L. } \\
\text { (Amaryllidaceae) }\end{array}$ & $\begin{array}{l}\text { Aqueous } \\
\text { (bulbs) }\end{array}$ & $\begin{array}{c}\text { Significant increase in biochemical } \\
\text { parameters at } 100 \mathrm{mg} / \mathrm{kg}, \mathrm{p} .0 . \text { in } \\
\text { Wistar albino rats } \\
\text { W0] }\end{array}$ & Aqueous (bulbs) & $\begin{array}{c}\text { MIC } 1.95 \mu \mathrm{g} / \mathrm{ml} \text { using } \\
\text { REMA }^{[72]}\end{array}$ \\
\hline $\begin{array}{c}\text { Andrographis } \\
\text { paniculata (Burm.f.) } \\
\text { Nees (Acanthaceae) }\end{array}$ & $\begin{array}{c}\text { Ethanol } \\
\text { (whole plant) }\end{array}$ & $\begin{array}{l}\text { Significant increase in biochemical } \\
\text { parameters at } 25 \mathrm{mg} / \mathrm{kg}, \text { p.o. in } \\
\text { Balb/c mice }{ }^{[81]}\end{array}$ & $\begin{array}{c}\text { Aqueous } \\
\text { (whole plant) }\end{array}$ & $\begin{array}{l}100 \% \text { inhibition at } \\
5 \mathrm{mg} / \mathrm{ml} \text { using } \\
\text { Lowenstein-Jensen } \\
\text { proportion method }{ }^{[82]}\end{array}$ \\
\hline $\begin{array}{l}\text { Calophyllum } \\
\text { brasiliense Cambess. } \\
\text { (Clusiaceae) }\end{array}$ & $\begin{array}{l}\text { Methanol } \\
\text { (roots) }\end{array}$ & $\begin{array}{l}\text { Significant proliferation at } 200 \mu \mathrm{g} / \\
\mathrm{ml} \text { in splenocytes }\end{array}$ & $\begin{array}{l}\text { Dichloromethane and } \\
\text { aqueous (leaves) }\end{array}$ & $\begin{array}{c}\text { MIC } 125 \mu \mathrm{g} / \mathrm{ml} \text { using } \\
\text { REMA }^{[84]}\end{array}$ \\
\hline $\begin{array}{l}\text { Centella asiatica } \\
\text { (L.) Urb. (Apiaceae) }\end{array}$ & $\begin{array}{c}\text { Aqueous and } \\
\text { ethanol (whole } \\
\text { plant) }\end{array}$ & $\begin{array}{l}\text { Significant increase in PBMCs } \\
\text { proliferation and IL-2 production at } \\
500 \mu \mathrm{g} / \mathrm{ml}^{[85]}\end{array}$ & $\begin{array}{l}\text { Aqueous (whole } \\
\text { plant) }\end{array}$ & $\begin{array}{l}78.5 \% \text { inhibition at } 5 \\
\text { mg/ml using Lowenstein- } \\
\text { Jensen proportion } \\
\text { method }^{[82]}\end{array}$ \\
\hline $\begin{array}{c}\text { Glycyrrhiza } \\
\text { glabra L. } \\
\text { (Leguminosae) }\end{array}$ & Aqueous (roots) & $\begin{array}{l}\text { Significant increase in biochemical } \\
\text { parameters at } 1500 \mathrm{mg} / \mathrm{kg}, \mathrm{p} .0 . \text {, in } \\
\text { Swiss albino mice }{ }^{[86]}\end{array}$ & Ethanol (roots) & $\begin{array}{l}\text { MIC } 500 \mu \mathrm{g} / \mathrm{ml} \\
\text { using BACTEC } 460 \\
\text { radiorespirometric } \\
\text { assay }^{[87]}\end{array}$ \\
\hline $\begin{array}{l}\text { Adhatoda vasica L. } \\
\text { (Acanthaceae) }\end{array}$ & $\begin{array}{l}\text { Methanol, } \\
\text { chloroform and } \\
\text { diethyl ether } \\
\text { (leaves) }\end{array}$ & $\begin{array}{l}\text { Significant increase in biochemical } \\
\text { parameters at } 400 \mathrm{mg} / \mathrm{kg}, \mathrm{p} .0 . \text { in } \\
\text { Wistar albino rats }\end{array}$ & Aqueous (leaves) & $\begin{array}{l}70 \% \text { inhibition at } 4 \% \\
\text { using Lowenstein-Jensen } \\
\text { proportion method }{ }^{[57]}\end{array}$ \\
\hline $\begin{array}{l}\text { Ocimum basilicum L. } \\
\text { (Lamiaceae) }\end{array}$ & $\begin{array}{l}\text { Aqueous } \\
\text { and ethanol } \\
\text { (leaves) }\end{array}$ & $\begin{array}{l}\text { Significant increase in biochemical } \\
\text { parameters at } 400 \mathrm{mg} / \mathrm{kg}, \mathrm{p} .0 \text {. in } \\
\text { Swiss albino mice }{ }^{[89]}\end{array}$ & $\begin{array}{l}\text { 80\% methanol } \\
\text { (seeds) }\end{array}$ & $\begin{array}{c}\text { MIC } 25 \mu g / m l \text { using } \\
\text { REMA }^{[68]}\end{array}$ \\
\hline $\begin{array}{c}\text { Stachytarpheta } \\
\text { cayennensis (Rich.) } \\
\text { Vahl (Verbenaceae) }\end{array}$ & $\begin{array}{l}\text { Methanol } \\
\text { (leaves) }\end{array}$ & $\begin{array}{c}\text { 139.64\% phagocytic stimulation at } \\
100 \mu \mathrm{g} / \mathrm{ml} \text { in neutrophils }{ }^{[90]}\end{array}$ & $\begin{array}{l}\text { Aqueous (leaves and } \\
\text { roots) }\end{array}$ & $\begin{array}{c}M I C>200 \mu \mathrm{g} / \mathrm{ml} \text { using } \\
\mathrm{REMA}^{[91]}\end{array}$ \\
\hline $\begin{array}{l}\text { Thymus vulgaris L. } \\
\text { (Lamiaceae) }\end{array}$ & $\begin{array}{c}\text { Aqueous and } \\
\text { hexane (aerial) }\end{array}$ & $\begin{array}{l}\text { Significant decrease in splenocytes } \\
\text { proliferation at } 100 \mu \mathrm{g} / \mathrm{m}^{[92,93]}\end{array}$ & $\begin{array}{l}\text { Acetone and aqueous } \\
\text { (aerial) }\end{array}$ & $\begin{array}{l}\text { MIC } 0.5-5 \mathrm{mg} / \mathrm{ml} \text { by the } \\
\text { agar plate method }{ }^{[94]}\end{array}$ \\
\hline $\begin{array}{l}\text { Withania } \\
\text { somnifera (L.) Dunal } \\
\text { (Solanaceae) }\end{array}$ & $\begin{array}{l}70 \% \text { methanol } \\
\text { (roots) }\end{array}$ & $\begin{array}{c}\text { Significant increase in biochemical } \\
\text { parameters at } 20 \mathrm{mg} \text {, i.p., in } \\
\text { Balb/c mice }{ }^{[95]}\end{array}$ & Aqueous (leaves) & $\begin{array}{c}\text { MIC } 1 \mathrm{mg} / \mathrm{ml} \text { with } \\
64.47 \% \text { inhibition by } \\
\text { proportion and absolute } \\
\text { concentration method }{ }^{[96]}\end{array}$ \\
\hline
\end{tabular}


TABLE 4: PHYTOCHEMICAL CONSTITUENTS WITH IMMUNOMODULATORY AND ANTIMYCOBACTERIAL ACTIVITIES

\begin{tabular}{|c|c|c|c|c|c|}
\hline \multicolumn{2}{|c|}{ Phytochemical constituents } & \multicolumn{2}{|r|}{ Immunomodulatory activity } & \multicolumn{2}{|c|}{ Antimycobacterial activity } \\
\hline Group & Name & Source & Results & Source & $M I C(\mu \mathrm{g} / \mathrm{ml})$ \\
\hline \multirow[t]{2}{*}{ Alkaloid } & Piperine & $\begin{array}{l}\text { Pure standard } \\
\text { preclinical dose }\end{array}$ & $\begin{array}{l}\text { Significant increase in the leucocytes in } \\
\text { Swiss albino mice at } 100 \mathrm{mg} / \mathrm{kg}^{[97]}\end{array}$ & $\begin{array}{c}\text { Pure standard } \\
\text { preclinical dose } 1 \\
\mathrm{mg} / \mathrm{kg}^{[98]}\end{array}$ & -- \\
\hline & Quercetin & $\begin{array}{l}\text { Urtica dioica } \\
\text { aerial parts }\end{array}$ & $\begin{array}{l}\text { Enhanced stimulation of neutrophils at } \\
\qquad 16 \mu \mathrm{g}^{[99]}\end{array}$ & $\begin{array}{l}\text { Tussilago farfara } \\
\text { aerial parts } \\
\end{array}$ & 500 \\
\hline Flavonoids & Genistein & $\begin{array}{l}\text { Pueraria tuberosa } \\
\text { tubers }\end{array}$ & $\begin{array}{l}\text { Significant decrease in the leucocytes } \\
\text { and phagocytic index in Swiss albino rats } \\
\text { at } 25 \mathrm{mg} / \mathrm{kg}, \text { p.o. }^{[101]}\end{array}$ & $\begin{array}{l}\text { Ficus nervosa } \\
\text { roots }^{[102]}\end{array}$ & 35 \\
\hline Quinone & Plumbagin & $\begin{array}{l}\text { Plumbago } \\
\text { zeylanica roots }\end{array}$ & $\begin{array}{l}\text { Significant modulation of T-cell } \\
\text { proliferation and cytokine response in } \\
\text { DBA/1 mice at } 3.3 \mathrm{mg} / \mathrm{kg} \text { i.p.p. }{ }^{[103]}\end{array}$ & $\begin{array}{l}\text { Diospyros } \\
\text { anisandra stem } \\
\text { bark }^{[104]}\end{array}$ & 12.5 \\
\hline \multirow[t]{3}{*}{ Phenolics } & Caffeic acid & $\begin{array}{l}\text { Propolis (bee } \\
\text { glue) }\end{array}$ & $\begin{array}{l}\text { Significant proliferation of leucocytes at } \\
50 \mathrm{mg} / \mathrm{kg} \text { in CBA mice }{ }^{[105]}\end{array}$ & $\begin{array}{l}\text { Tussilago farfara } \\
\text { aerial parts } \\
{[100]}\end{array}$ & 250 \\
\hline & Lupeol & $\begin{array}{l}\text { Gentiana kurroo } \\
\text { roots }\end{array}$ & $\begin{array}{l}\text { Significant increase in antibody titre in } \\
\text { Swiss albino mice at } 10 \mathrm{mg} / \mathrm{kg}^{[106]}\end{array}$ & $\begin{array}{l}\text { Zanthoxylum } \\
\text { capense roots }\end{array}$ & 25 \\
\hline & Ursolic acid & $\begin{array}{l}\text { Eucalyptus } \\
\text { tereticornis } \\
\text { leaves }\end{array}$ & $\begin{array}{l}\text { Significant increase in antibody titre in } \\
\text { Swiss albino mice at } 1 \mathrm{mg} / \mathrm{kg}^{[106]}\end{array}$ & $\begin{array}{l}\text { Lantana hispida } \\
\text { aerial parts } \\
\end{array}$ & 25 \\
\hline \multirow[t]{4}{*}{ Terpenoids } & Oleanolic acid & Pure standard & $\begin{array}{c}\text { Significant expression of IFN-y and } \\
\text { TNF- } \alpha \text { in lungs of BALB/c mice at } 5 \mathrm{mg} / \\
\mathrm{kg} \text {, s.c. }{ }^{[109]}\end{array}$ & $\begin{array}{l}\text { Lantana hispida } \\
\text { aerial parts }{ }^{[108]}\end{array}$ & 50 \\
\hline & Stigmasterol & Pure standard & $\begin{array}{l}\text { Inhibition of inflammatory mediators } \\
\text { at } 20 \mu \mathrm{g} / \mathrm{ml}^{[110]}\end{array}$ & $\begin{array}{l}\text { Morinda citrifolia } \\
\quad \text { leaves [111] }\end{array}$ & 32 \\
\hline & B-sitosterol & $\begin{array}{l}\text { Cordia rothii } \\
\quad \text { leaves }\end{array}$ & $\begin{array}{l}\text { Significant increase in inflammatory } \\
\text { mediators of neutrophils and T-cell } \\
\text { proliferation at } 100 \mu \mathrm{g} / \mathrm{ml}^{[112]}\end{array}$ & $\begin{array}{l}\text { Morinda citrifolia } \\
\text { leaves }^{[111]}\end{array}$ & 128 \\
\hline & Betulinic acid & $\begin{array}{l}\text { Euphorbia } \\
\text { spinidens aerial } \\
\text { parts }\end{array}$ & Stimulation of PBMCs at $50 \mu \mathrm{g} / \mathrm{ml}^{[113]}$ & $\begin{array}{l}\text { Valeriana } \\
\text { laxiflora aerial } \\
\text { parts }^{[114]}\end{array}$ & 62.5 \\
\hline
\end{tabular}

potential ${ }^{[81]}$. In an another study, the aqueous extract of $A$. paniculata exhibited $100 \%$ inhibition against M. tuberculosis $\mathrm{H} 37 \mathrm{Rv}$ strain at $5 \mathrm{mg} / \mathrm{ml}$ using Lowenstein-Jensen proportion method suggesting its antimycobacterial potential ${ }^{[82]}$. Methanol extract of Calophyllum brasiliense roots has been reported to significantly stimulate proliferation of splenocytes from mice at $200 \mu \mathrm{g} / \mathrm{ml}$ using in vitro cellular proliferation assay ${ }^{[83]}$. Antimycobacterial activity of the dichloromethane and aqueous extracts prepared from Calophyllum brasiliense leaves was reported with MIC values of 62.5 and $125 \mu \mathrm{g} / \mathrm{ml}$ against $M$. tuberculosis H37Rv strain, respectively using resazurin microtiter $\operatorname{assay}^{[84]}$.

Punturee et al. reported that the aqueous and ethanol extracts of Centella asiatica whole plant significantly increased production of IL-2 and TNF- $\alpha$ in human peripheral blood mononuclear cells at $500 \mu \mathrm{g} / \mathrm{ml}$. In addition, it also showed greater response to both primary and secondary antibodies in BALB/c mice at $100 \mathrm{mg} / \mathrm{kg}$, which indicated a promising effect on non- specific cellular and humoral immune system ${ }^{[85]}$. The aqueous extract of Centella asiatica whole plant showed antiTB activity at $5 \mathrm{mg} / \mathrm{ml}$ with $78.5 \%$ inhibition of M. tuberculosis H37Rv strain using Lowenstein-Jensen proportion method ${ }^{[82]}$. Administration of the aqueous root extract of Glycyrrhiza glabra exhibited significant increase in the biochemical parameters at $1500 \mathrm{mg} / \mathrm{kg}$ in Swiss albinomice, which suggested immunomodulatory potential with respect to cellular immunity, phagocytic response and anaphylactic reaction ${ }^{[86]}$. In another study on the ethanol extract of Glycyrrhiza glabra root, significant antimycobacterial activity was observed against $M$. tuberculosis $\mathrm{H} 37 \mathrm{Rv}$ strain at $500 \mu \mathrm{g} / \mathrm{ml}$ using BACTEC 460 radiorespirometric assay ${ }^{[87]}$. A study reported by Vinothapooshan and Sundar validated the immunomodulatory properties of methanol, chloroform and diethyl ether extracts of Adhatoda vasica leaves in experimental animals. Oral administration of the extracts at $400 \mathrm{mg} / \mathrm{kg}$ in Wistar rats significantly increased the neutrophil adhesion to nylon fiber and induced delayed type hypersensitivity reaction to sheep erythrocytes ${ }^{[88]}$. While in other 
study, the aqueous extract of $A$. vasica leaves showed $70 \%$ inhibition of $M$. tuberculosis $\mathrm{H} 37 \mathrm{Rv}$ strain at $4 \% \mathrm{v} / \mathrm{v}$ in Lowenstein-Jensen proportion method ${ }^{[57]}$. Oral administration of the aqueous and ethanol extract of Ocimum basilicum leaves at $400 \mathrm{mg} / \mathrm{kg}$ showed a significant increase in the production of circulating antibody titer, delayed type hypersensitivity reaction in sheep erythrocytes sensitized mice ${ }^{[89]}$. In another study, $80 \%$ methanol extract of $O$. basilicum seeds exhibited antimycobacterial activity against $M$. tuberculosis strains with the MIC value $25 \mu \mathrm{g} / \mathrm{ml}$ using resazurin microtitre assay ${ }^{[68]}$.

Methanol extract of Stachytarpheta cayennensis leaves showed $139.64 \%$ phagocytic stimulation at $100 \mu \mathrm{g} / \mathrm{ml}$ in neutrophils suggesting its immunostimulatory action ${ }^{[90]}$. Carrion et al. reported the antimycobacterial activity of the aqueous extract of $S$. cayennensis leaves and roots with the MIC value greater than $200 \mu \mathrm{g} / \mathrm{ml}$ using resazurin microtitre assay ${ }^{[91]}$. A study on the aqueous and hexane extract of Thymus vulgaris aerial parts reported significant decrease in the number of splenocytes at $100 \mu \mathrm{g} / \mathrm{ml}$ indicating potent immunomodulatory activity ${ }^{[92,93]}$. Whereas, acetone and aqueous extract of $T$. vulgaris aerial parts inhibited the growth of M. tuberculosis showing the MIC value 0.5$5 \mathrm{mg} / \mathrm{ml}$ by the agar plate method ${ }^{[94]}$. Administration of $70 \%$ methanol extract of Withania somnifera roots exhibited significant increase in the total leucocytes count, bone marrow cellularity and also produced an enhancement in the circulating antibody titre, number of plaque forming cells and phagocytic activity of peritoneal macrophages at $20 \mathrm{mg}$ in Balb/c mice ${ }^{[95]}$. Adaikkappan et al. reported the antimycobacterial activity of the aqueous extract of $W$. somnifera leaves with MIC value $1 \mathrm{mg} / \mathrm{ml}$ and $64.47 \%$ inhibition of M. tuberculosis $\mathrm{H} 37 \mathrm{Rv}$ (MTB) strain by proportion and absolute concentration method ${ }^{[96]}$. These reports clearly suggested that these plants are rich source of phytochemical constituents with immunomodulatory and antimycobacterial potential.

Piperine $\left(\mathrm{C}_{17} \mathrm{H}_{19} \mathrm{NO}_{3}\right)$, an alkaloid significantly reduced the toxicity of allethrin in Swiss albino mice at $100 \mathrm{mg} / \mathrm{kg}$ by increasing the number of leucocytes ${ }^{[97]}$. Additionally, it exhibited proliferation of $\mathrm{T}$ and $\mathrm{B}$ cells and enhanced macrophage activation at $1 \mu \mathrm{g} / \mathrm{ml}$ concentration in the splenocytes of $\mathrm{BALB} / \mathrm{c}$ mice ${ }^{[98]}$. In murine model of MTB, combination of piperine and rifampicin $(1 \mathrm{mg} / \mathrm{kg})$ exhibited better efficacy and resulted in 1.4 to $0.8 \log$ reduction in the bacterial $\operatorname{load}^{[98]}$. Thus, piperine can be used as an adjunct therapy in the management of TB. Flavonoids, a large group of polyphenolic compounds having a benzopyrone structure are ubiquitously present in plants. Quercetin $\left(\mathrm{C}_{15} \mathrm{H}_{10} \mathrm{O}_{7}\right)$, the major compound of the methanol extract of the aerial parts of Urtica dioica L. enhanced the stimulation of neutrophils at $16 \mu \mathrm{g}$ dose in an in vitro experiment ${ }^{[99]}$. On the other hand, quercetin isolated from the aerial parts of Tussilago farfara showed antitubercular activity against MTB H37Rv strain using a high throughput spot culture growth inhibition assay ${ }^{[100]}$. In another study, Maji et al. reported that the administration of genistein $\left(\mathrm{C}_{15} \mathrm{H}_{10} \mathrm{O}_{5}\right)$ significantly modulated the innate as well as humoral immune responses against SRBCs challenged Swiss albino rats at a dose of $25 \mathrm{mg} / \mathrm{kg}^{[101]}$. Amongst the various coumarins and flavonoids isolated from the ethyl acetate extract of Ficus nervosa roots, genistein showed the antimycobacterial activity against MTB $\mathrm{H} 37 \mathrm{Rv}^{[102]}$. Plumbagin $\left(\mathrm{C}_{11} \mathrm{H}_{8} \mathrm{O}_{3}\right)$, a naphthoquinone found in the roots of Plumbago zeylanica showed significant modulation of $\mathrm{T}$-cell proliferation and cytokine response in DBA/1 mice at $3.3 \mathrm{mg} / \mathrm{kg}^{[103]}$. This study demonstrated the novel therapeutic role of plumbagin in the pathogenesis of rheumatoid arthritis. In addition, plumbagin isolated from the hexane fraction of the stem bark of Diospyros anisandra showed the strongest antimycobacterial activity against the resistant strain ${ }^{[104]}$.

Caffeic acid $\left(\mathrm{C}_{9} \mathrm{H}_{8} \mathrm{O}_{4}\right)$, a polyphenolic compound from propolis exhibited significant proliferation ofleukocytes at a dose of $50 \mathrm{mg} / \mathrm{kg}$ in CBA mice and demonstrated a modulatory activity on the macrophages ${ }^{[105]}$. In another study, trans-caffeic acid isolated from the aerial parts of Tussilago farfara showed antitubercular activity against MTB H37Rv strain using a high throughput spot culture growth inhibition assay ${ }^{[100]}$. Maurya et al. reported that the administration of two purified triterpenoids; lupeol $\left(\mathrm{C}_{30} \mathrm{H}_{50} \mathrm{O}\right)$ isolated from Gentiana kurroo roots and ursolic acid $\left(\mathrm{C}_{30} \mathrm{H}_{48} \mathrm{O}_{3}\right)$ isolated from Eucalyptus tereticornis leaves, exhibited significant increase in the antibody titer in rabbit red blood cells immunized Swiss albino mice at doses of 10 and $1 \mathrm{mg} / \mathrm{kg}$, respectively ${ }^{[106]}$. Also, lupeol isolated from Zanthoxylum capense roots ${ }^{[107]}$ and ursolic acid from Lantana hispida aerial parts ${ }^{[108]}$ showed ability to inhibit the growth of M. tuberculosis H37Rv indicting potential antimycobacterial activity. Oleanolic acid $\left(\mathrm{C}_{30} \mathrm{H}_{48} \mathrm{O}_{3}\right)$, another pentacyclic triterpenoid compound 
exhibited significant expression of IFN- $\gamma$ and TNF- $\alpha$ in the lungs of BALB/c mice at $5 \mathrm{mg} / \mathrm{kg}^{[109]}$. On the other hand, oleanolic acid isolated from Lantana hispida aerial parts showed antitubercular activity against MTB $\mathrm{H} 37 \mathrm{Rv}$ with a MIC value of $50 \mu \mathrm{g} / \mathrm{ml}^{[108]}$. Stigmasterol $\left(\mathrm{C}_{29} \mathrm{H}_{48} \mathrm{O}\right)$, a plant sterol has been reported to inhibit the inflammatory mediators at $20 \mu \mathrm{g} / \mathrm{ml}^{[110]}$. In another study, stigmasterol isolated from the hexane fraction of Morinda citrifolia leaves showed antimycobacterial activity with MIC value $32 \mu \mathrm{g} / \mathrm{ml}^{[111]}$. $\beta$-sitosterol $\left(\mathrm{C}_{29} \mathrm{H}_{50} \mathrm{O}\right)$, a phytosterol isolated from Cordia rothii leaves has also been evaluated for immunomodulatory activity and was found to increase significantly the number of neutrophils and T-cells at $100 \mu \mathrm{g} / \mathrm{ml}^{[112]}$. Also, $\beta$-sitosterol isolated from the hexane fraction of $M$. citrifolia leaves showed antimycobacterial activity with an MIC value of $32 \mu \mathrm{g} / \mathrm{ml}^{[111]}$. A study reported by Ghannadian et al. suggests that betulinic acid $\left(\mathrm{C}_{30} \mathrm{H}_{48} \mathrm{O}_{3}\right)$, a pentacyclic triterpenoid isolated from Euphorbia spinidens aerial parts resulted in the stimulation of lymphocyte proliferation at $50 \mu \mathrm{g} / \mathrm{ml}^{[113]}$. Moreover, betulinic acid isolated from Valeriana laxiflora aerial parts also showed antitubercular activity against $M$. tuberculosis with MIC value of $62.5 \mu \mathrm{g} / \mathrm{ml}^{[114]}$. Thus, the phytochemical constituents like terpenoids, phenolics, flavonoids, quinones possess both immunomodulatory and antimycobacterial potential.

As globally around 2 million people die annually due to $\mathrm{TB}$, this review could be of help to the scientists making attempts to discover novel natural products to develop as new antiTB drugs. There are many active plant extracts from which active compounds are yet to be isolated. It is necessary to make efforts to identify and characterize the active constituents from medicinal plants. In addition, drugs acting synergistically could be developed from plant extracts. Promising leads from plant sources might also act on newer targets and thus, could play a crucial role in the development of new generation of antiTB drugs. For eradicating TB, instead of focusing on a single drug that hits a single target, the approach of systems biology should be followed using multiple compounds that hit multiple targets in different pathways.

\section{Acknowledgements:}

Authors acknowledge the assistance from the Board of Research in Nuclear Sciences, Government of India (Sanction no. 2010/37B/37/BRNS).

\section{Conflict of interest:}

The authors declare that they have no conflict of interest.

\section{REFERENCES}

1. Dubey D, Rath S, Sahu MC, Debata NK, Padhy RN. Antimicrobials of plant origin against TB and other infections and economics of plant drugs-Introspection. Indian $\mathrm{J}$ Tradit Know 2012;11:225-33.

2. Smith NH, Gordon SV, de la Rua-Domenech R, CliftonHadley RS, Hewinson RG. Bottlenecks and broomsticks: the molecular evolution of Mycobacterium bovis. Nat Rev Microbiol 2006;4:670-81.

3. de Kantor IN, LoBue PA, Thoen CO. Human tuberculosis caused by Mycobacterium bovis in the United States, Latin America and the Caribbean. Int J Tuberc Lung Dis 2010;14(11):1369-73.

4. Jordao L, Vieira OV. Tuberculosis: new aspects of an old disease. Int J Cell Biol 2011;2011:403623.

5. de Jong BCD, Antonio M, Gagneux S. Mycobacterium africanum-Review of an important cause of human tuberculosis in West Africa. PLoS Negl Trop Dis 2010;4(9):e744.

6. Zumla A, Raviglione M, Hafner R, von Reyn CF. Tuberculosis. N Engl J Med 2013;368(8):745-55.

7. Ibekwe NN, Ameh SJ. Plant natural products research in tuberculosis drug discovery and development: A situation report with focus on Nigerian biodiversity. Afr J Biotechnol 2014;13:2307-20.

8. Sharma SK, Mohan A. Tuberculosis: From an incurable scourge to a curable disease - journey over a millennium. Indian J Med Res 2013;137(3):455-93.

9. Waksman SA. The conquest of tuberculosis. Berkeley and Los Angeles: University of California Press; 1964.

10. Keers RY. Pulmonary tuberculosis - A journey down the centuries. London: Bailliere-Tindall; 1978.

11. BhatchaMK. Review onherbaldrug forTB/ethnopharmacology of tuberculosis. Int J Pharma Res 2013;1:1-8.

12. Zink A, Sola C, Reischl U, Grabner W, Rastogi N, Wolf H, et al. Characterization of Mycobacterium tuberculosis Complex DNAs from Egyptian Mummies by Spoligotyping. J Clin Microbiol 2003;41:359-67.

13. Global tuberculosis report 2016. WHO, World Health Organization. Available from: http://apps.who.int/ medicinedocs/en/d/Js23098en/.

14. TB India 2014. Annual Status Report. Revised National TB Control Programme. Central TB Division. Directorate General of Health Services, New Delhi. Available from: https://tbcindia.gov.in/showfile.php?lid=3142.

15. Bhargava A, Benedetti A, Oxlade O, Pai M, Menzies D. Undernutrition and the incidence of tuberculosis in India: National and sub-national estimates of the population attributable fraction related to under-nutrition. Natl Med J India 2014;27(3):4-9.

16. Manikandan S. Treating tuberculosis: Time to introduce fixeddose drug combinations. J Young Pharm 2012;4(4):199-200.

17. Ahmad S. Pathogenesis, immunology and diagnosis of latent Mycobacterium tuberculosis infection. Clin Dev Immunol 2011;2011:814943.

18. Dartois V. The path of antituberculosis drugs: from blood 
to lesions to mycobacterial cells. Nat Rev Microbiol 2014;12(3):159-67.

19. Cooper AM. Cell-mediated immune responses in tuberculosis. Annu Rev Immunol 2009;27:393-422.

20. Dutta NK, Karakousis PC. Latent tuberculosis infection: myths, models, and molecular mechanisms. Microbiol Mol Biol Rev 2014;78(3):343-71.

21. Kaplan G, Post FA, Moreira AL, Wainwright H, Kreiswirth $\mathrm{BN}$, Tanverdi M, et al. Mycobacterium tuberculosis growth at the cavity surface: a microenvironment with failed immunity. Infect Immun 2003;71(12):7099-108.

22. Ulrichs T, Kaufmann SH. New insights into the function of granulomas in human tuberculosis. J Pathol 2006;208(2):261-9.

23. Bozzano F, Marras F, Maria AD. Immunology of tuberculosis. Mediterr J Hematol Infect Dis 2014;6(1):e2014027.

24. Rieder HL, Chen-Yuan C, Gie RP, Enarson DA. Clinical Tuberculosis. International Union against Tuberculosis and Lung Diseases. 3rd ed. Oxford: Macmillan Education; 2009.

25. Borgdorff MW, Nagelkerke NJD, de Haas PEW, van Soolingen D. Transmission of Mycobacterium tuberculosis depending on the age and sex of source cases. Am J Epidemiol 2001;154:934-43.

26. Hsu DC, Katelaris CH. Long-term management of patients taking immunosuppressive drugs. Aust Prescr 2009;32:68-71.

27. Oxlade O, Murray M. Tuberculosis and poverty: Why are the poor at greater risk in India? PLOS One 2012;7(11);e47533.

28. Katoch VM. Newer diagnostic techniques for tuberculosis. Indian J Med Res 2004;120(4):418-28.

29. Steingart KR, Ramsay A, Dowdy D, Pai M. Serological tests for the diagnosis of active tuberculosis: relevance for India. Indian J Med Res 2012;135(5):695-702.

30. Riccardi G, Pasca MR. Trends in discovery of new drugs for tuberculosis therapy. J Antibiot 2014;67:655-9.

31. Zumla AI, Gillespie SH, Hoelscher M, Philips PPJ, Cole ST, Abubakar I, et al. New antituberculosis drugs, regimens, and adjunct therapies: needs, advances, and future prospects. Lancet Infect Dis 2014;14(4):327-40.

32. Zumla A, Nahid P, Cole ST. Advances in the development of new tuberculosis drugs and treatment regimens. Nat Rev Drug Discov 2013;12(5):388-404.

33. Goldberg DE, Siliciano RF, Jacobs Jr WR. Outwitting evolution: Fighting drug-resistant TB, Malaria, and HIV. Cell 2012;148(6):1271-83.

34. Davies PD. The role of DOTS in tuberculosis treatment and control. Am J Respir Med 2003;2(3):203-9.

35. Awofeso N. Anti-tuberculosis medication side-effects constitute major factor for poor adherence to tuberculosis treatment. Bull World Health Organ 2008;86(3):B-D.

36. Torun T, Gungor G, Ozmen I, Bolukba Y, Maden E, Bicakci $\mathrm{B}$, et al. Side effects associated with the treatment of multidrugresistant tuberculosis. Int J Tuberc Lung Dis 2005;9(12):1373-7.

37. Chhabra N, Aseri ML, Dixit R, Gaur S. Pharmacotherapy for multidrug resistant tuberculosis. J Pharmacol Pharmacother 2012;3(2):98-104.

38. Garner P, Smith H, Munro S, Volmink J. Promoting adherence to tuberculosis treatment. Bull World Health Organ 2007;85(5):404-6.

39. Adhvaryu M, Vakharia B. Drug-resistant tuberculosis: emerging treatment options. Clin Pharmacol 2011;3:51-67.

40. Palomino JC, Martin A. Drug resistance mechanisms in Mycobacterium tuberculosis. Antibiotics 2014;3(3):317-40.

41. Grange JM, Brunet LR, Rieder HL. Immune protection against tuberculosis - When is immunotherapy preferable to vaccination? Tuberculosis 2011;91(2):179-85.

42. Uhlin M, Andersson J, Zumla A, Maeurer M. Adjunct Immunotherapies for Tuberculosis. $J$ Infect Dis 2012;205Supp12:S325-34.

43. Bourinbair AS, Mezentseva MV, Butov DA, Nyasulu PS, Efremenko YV, Jirathitikal V, et al. Immune approaches in tuberculosis therapy: a brief overview. Expert Rev Anti Infect Ther 2012;10(3):381-9.

44. Expert consultation on immunotherapeutic interventions for tuberculosis. Report by World Health Organization. Available from: https://www.who.int/tdr/publications/tdr-researchpublications/immunotherapeutic-interventions-tb/en/.

45. Grange JM. Immunotherapy: A new strategy for tuberculosis control? Respir Med 1997;91(1):1-4.

46. Guo S, Zhao J. Immunotherapy for Tuberculosis: what's the better choice? Front Biosci 2012;17:2684-90.

47. Rivero-Lezcano OM. Cytokines as immunomodulators in tuberculosis therapy. Recent Pat Antiinfect Drug Discov 2008;3(3):168-76.

48. Yaseen NY, Thewaini AJ, Al-Tawil NG, Jazrawi FY. Trial of immunopotentiation by levamisole in patients with pulmonary tuberculosis. J Infect 1980;2(2):125-36.

49. Colizza S, Bagolan P, Paola MD. Side effects to levamisole given to neoplastic patients as adjuvant to surgery: A new case of agranulocytosis. J Surg Oncol 1981;16(3):259-64.

50. Ekambaram S, Mahalingam V, Nageswaran P, Udani A, Geminiganesan S, Priyadarshini S. Efficacy of levamisole in children with frequently relapsing and steroid-dependent nephrotic syndrome. Indian Pediatr 2014;51(5):371-3.

51. Casale TB, Stokes JR. Immunomodulators for allergic respiratory disorders. Clin Rev Allergy Immunol 2008;121(2):288-96.

52. Newton SM, Lau C, Gurcha SS, Besra GS, Wright CW. The evaluation of forty-three plant species for in vitro antimycobacterial activities; isolation of active constituents from Psoralea corylifolia and Sanguinaria canadensis. J Ethnopharmacol 2002;79(1):57-67.

53. Bhattacharya D, Dwivedi VP, Das G. Revisiting immunotherapy in tuberculosis. J Mycobac Dis 2013;4:e123.

54. Kuete V, Tabopda TK, Ngameni B, Nana F, Tshikalange TE, Ngadjui BT. Antimycobacterial, antibacterial and antifungal activities of Terminalia superba (Combretaceae). S Afr J Bot 2010;76:125-31.

55. Cowan MM. Plant products as antimicrobial agents. Clin Microbiol Rev 1999;12(4):564-82.

56. Debnath PK, Chattopadhyay J, Mitra A, Adhikari A, Alam MS, Bandopadhyay SK, et al. Adjunct therapy of Ayurvedic medicine with anti-tubercular drugs on the therapeutic management of pulmonary tuberculosis. J Ayurveda Integr Med 2012;3(3):141-9.

57. Gupta R, Thakur B, Singh P, Singh HB, Sharma VD, Katoch $\mathrm{VM}$, et al. Anti-tuberculosis activity of selected medicinal plants against multi-drug resistant Mycobacterium tuberculosis isolates. Indian J Med Res 2010;131:809-13.

58. Ashenafi S, Aderaye G, Bekele A, Zewdie M, Aseffa G, Hoang AT, et al. Progression of clinical tuberculosis is associated with a Th2 immune response signature in combination with elevated levels of SOCS3. Clin Immunol 2014;151(2):84-99.

59. Romani L, Puccetti P, Bistoni F. Interleukin-12 in infectious diseases. Clin Microbiol Rev 1997;10:611-36. 
60. Mishra LC. Scientific basis for Ayurvedic Therapies. Florida: CRC Press LLC; 2004.

61. Mahima, Rahal A, Deb R, Latheef SK, Samad HA, Tiwari R, et al. Immunomodulatory and therapeutic potentials of herbal, traditional/indigenous and ethnoveternary medicines. Pak J Biol Sci 2012;15(16):754-74.

62. Park IJ, Cha SY, Kang M, Jang HK. Immunomodulatory effect of a proanthocyanidin-rich extract from Pinus radiata bark by dosing period in chickens. Poult Sci 2013;92(2):352-7.

63. Talmale S, Bhujade A, Patil M. Immunostimulatory activity of flavonoids isolated from stem bark of Zizyphus mauritiana. IJIRSET 2014;3:14285-96.

64. Mukherjee PK, Nema NK, Bhadra S, Mukherjee D, Braga F, Matsabisa MG. Immunomodulatory leads from medicinal plants. Indian J Tradit Know 2014;13:235-56.

65. Devasagayam TBA, Saims KB. Immunostimulants and antioxidants from Indian medicinal plants. Indian J Exp Biol 2002;40:639-55.

66. Chiang LC, Ng LT, Chiang W, Chang MY, Lin CC. Immunomodulatory activities of flavonoids, monoterpenoids, triterpenoids, iridoid glyosides and phenolic compounds of Plantago species. Planta Med 2003;69(7):600-4.

67. Gemechu A, Giday M, Worku A, Ameni G. In vitro antimycobacterial activity of selected medicinal plants against Mycobacterium tuberculosis and Mycobacterium bovis strains. BMC Complement Altern Med 2013;13:291.

68. Balcha E, Mengiste B, Gebrelibanos M, Worku A, Ameni G. Evaluation of in vitro antimycobacterial activity of selected medicinal plants in Mekelle, Ethiopia. World Appl Sci J 2014;31:1217-20.

69. Sharma D, Yadav P, Jaya. An Overview of Phytotherapeutic Approaches for the Treatment of Tuberculosis. Mini Rev Med Chem 2017;17(2):163-83.

70. Ramachandran SS, Balasubramanian S. Plants: A source for new antimycobacterial drugs. Planta Med 2014;80(1):9-21.

71. Ramos DF, Leitao GG, Costa FN, Abreu L, Villarreal JV, Leitao SG, et al. Investigation of the antimycobacterial activity of 36 plant extracts from the Brazilian Atlantic forest. Braz J Pharm Sci 2008;44:669-74.

72. Viswanathan V, Phadatare AG, Mukne A. Antimycobacterial and antibacterial activity of Allium sativum bulbs. Indian $\mathrm{J}$ Pharm Sci 2014;76(3):256-61.

73. Gautam R, Saklani A, Jachak SM. Indian medicinal plants as a source of antimycobacterial agents. J Ethnopharmacol 2007;110(2):200-34.

74. Okunade AL, Elvin-Lewis MPF, Lewis WH. Natural antimycobacterial metabolites: current status. Phytochemistry 2004;65(8):1017-32.

75. Negi AS, Kumar JK, Luqman S, Saikia D, Khanuja SP. Antitubercular potential of plants: a brief account of some important molecules. Med Res Rev 2010;30(4):03-645.

76. Jimenez-Arellanes A, Luna-Herrera J, Cornejo-Garrido J, Lopez-Garcia S, Castro-Mussot ME, Meckes-Fischer $\mathrm{M}$, et al. Ursolic and oleanolic acids as antimicrobial and immunomodulatory compounds for tuberculosis treatment. BMC Complement Altern Med 2013;13:258.

77. Shailajan S, Menon S, Kulkarni S, Tiwari B. Standardized extract of Mangifera indica L. leaves as an antimycobacterial and immunomodulatory agent. Pharmcog Commn 2016;6:137-47.

78. Patel P, Asdaq SMB. Immunomodulatory activity of methanolic fruit extract of Aegle marmelos in experimental animals. Saudi Pharm J 2010;18(3):161-5.
79. Kaur R, Kaur H. Antitubercular activity and phytochemical screening of selected medicinal plants. Orient $\mathrm{J}$ Chem 2015;31:597-600.

80. Tende JA, Eze ED, Muhammad Z, Daikwo OA. Immunomodulatory activity of Garlic (Allium sativum) in Wistar Rats. Ann Exp Bio 2014;2:74-6.

81. Puri A, Saxena R, Saxena RP, Saxena KC, Srivastava V, Tandon JS. Immunostimulant agents from Andrographis paniculata. J Nat Prod 1993;56(7):995-9.

82. Radji M, Kurniati M, Kiranasari A. Comparative antimycobacterial activity of some Indonesian medicinal plants against multi-drug resistant Mycobacterium tuberculosis. J App Pharm Sci 2015;5:19-22.

83. Zandonai RH, Coelho F, Ferreira J, Mendes AKB, Biavatti MW, Niero R, et al. Evaluation of the proliferative activity of methanol extracts from six medicinal plants in murine spleen cells. Braz J Pharm Sci 2010;46(2):323-33.

84. Pires CT, Brenzan MA, Scodro RB, Cortez DA, Lopes LD, Siqueira VL, et al. Antimycobacterium tuberculosis activity and cytotoxicity of Calophyllum brasiliense Cambess (Clusiaceae). Mem Inst Oswaldo Cruz 2014;109(3):324-9.

85. Punturee K, Wild CP, Kasinrerk WK, Vinitketkumnuen U. Immunomodulatory activities of Centella asiatica and Rhinacanthus nasutus extracts. Asian Pac J Cancer Prev 2005;6(3):396-400.

86. Mazumder PM, Pattnayak S, Parvani H, Sasmal D, Rathinavelusamy P. Evaluation of immunomodulatory activity of Glycyrhiza glabra L roots in combination with zinc. Asian Pac J Trop Biomed 2012;2012:S15-S20.

87. Gupta VK, Fatima A, Faridi U, Negi AS, Shanker K, Kumar JK, et al. Antimicrobial potential of Glycyrrhiza glabra roots. J Ethnopharmacol 2008;116(2):377-80.

88. Vinothapooshan G, Sundar K. Immunomodulatory activity of various extracts of Adhatoda vasica Linn. in experimental rats. Afr J Pharma Pharmacol 2011;5:306-10.

89. Dashputre NL, Naikwade NS. Preliminary immunomodulatory activity of aqueous and ethanolic leaves extracts of Ocimum basilicum Linn in mice. Int J PharmTech Res 2010;2:1342-9.

90. Okoye TC, Akah PA, Ezike AC, Uzor PF, Odoh UE, Igboeme SO, et al. Immunomodulatory effects of Stachytarpheta cayennensis leaf extract and its synergistic effect with artesunate. BMC Complement Altern Med 2014;14:376.

91. Carrion LL, Ramos DF, Martins D, Osorio MIC, de Carvalho Cursino LM, Mesquita DWO, et al. Antimycobacterial activity of Brazillian Amazon plants extracts. Int J Phytomed 2013;5:479-85.

92. Amirghofran Z, Hashemzadeh R, Javidnia K, Golmoghaddam $\mathrm{H}$, Esmaeilbeig A. In vitro immunomodulatory effects of extracts from three plants of the Labiatae family and isolation of the active compound(s). J Immunotoxicol 2011;8(4):265-73.

93. Amirghofran Z, Ahmadi H, Karimi MH. Immunomodulatory activity of the water extracts of Thymus vulgaris, Thymus daenensis, and Zataria multiflora on dendritic cells and T cells responses. J Immunoassay Immunochem 2012;33(4):388-402.

94. Lall N, Meyer JJM. In vitro inhibition of drug-resistant and drug-sensitive strains of Mycobacterium tuberculosis by ethnobotanically selected South African plants. J Ethnopharmacol 1999;66(3):347-54.

95. Davis L, Kuttan G. Immunomodulatory activity of Withania somnifera. J Ethnopharmacol 2000;71(1-2):193-200.

96. Adaikkappan P, Kannapiran M, Anthonisamy A. Antimycobacterial activity of Withania somnifera and Pueraria 
tuberosa against Mycobacterium tuberculosis H37Rv. J Acad Indus Res 2012;1:153-6.

97. Divakar D, Sasmal D, Kumar A, Sharma N. Prophylactic Role of Piperine and Curcumin in Allethrin Altered Hematological and Biochemical Parameters in Swiss Albino Mice. Pharmacologia 2015;6:396-412.

98. Sharma S, Kalia NP, Suden P, Chauhan PS, Kumar M, Ram AB, et al. Protective efficacy of piperine against Mycobacterium tuberculosis. Tuberculosis 2014;94(4):389-96.

99. Akbay P, Basaran AA, Undeger U, Basaran N. In vitro immunomodulatory activity of flavonoid glycosides from Urtica dioica L. Phytother Res 2003;17(1):34-7.

100. Zhao J, Evangelopoulos D, Bhakta S, Gray AI, Seidel V. Antitubercular activity of Arctium lappa and Tussilago farfara extracts and constituents. J Ethnopharmacol 2014;155(1):796800.

101. Maji AK, Mahapatra S, Banerjee D. In vivo immunomodulatory potential of standardized Pueraria tuberosa extract and its isoflavonoids. Int J Pharm Pharm Sci 2014;6:861-7.

102. Chen LW, Cheng MJ, Peng CF, Chen IS. Secondary metabolites and antimycobacterial activities from the roots of Ficus nervosa. Chem Biodivers 2010;7(7):1814-21.

103. Poosarla A, Rao DN, Athota RR, Sunkara VG. Modulation of T-cell proliferation and cytokine response by Plumbagin, extracted from Plumbago zeylanica in collagen induced arthritis. BMC Complement Altern Med 2011;11:114.

104. Argaez RB, Chay CIC, Rodriguez LMP, Fernandez SS, Salinas GMM. Antimicrobial activity of Diospyros anisandra. Fitoterapia 2007;78:370-2.

105. Orsolic N, Knezevic AH, Sver L, Terzic S, Basic I. Immunomodulatory and antimetastatic action of propolis and related polyphenolic compounds. J Ethnopharmacol 2004;94(2-3):307-15.

106. Maurya A, Khan F, Bawankule DU, Yadav DK, Srivastava SK.
QSAR, docking and in vivo studies for immunomodulatory activity of isolated triterpenoids from Eucalyptus tereticornis and Gentiana kurroo. Eur J Pharm Sci 2012;47(1):152-61.

107. Luo X, Pires D, Ainsa JA, Gracia B, Duarte N, Mulhovo S, et al. Zanthoxylum capense constituents with antimycobacterial activity against Mycobacterium tuberculosis in vitro and ex vivo within human macrophages. J Ethnopharmacol 2013;146(1):417-22.

108. Jimenez-Arellanes A, Meckes M, Torres J, Luna-Herrera J. Antimycobacterial triterpenoids from Lantana hispida (Verbenaceae). J Ethnopharmacol 2007;111(2):202-5.

109. Jimenez A, Meckes M, Alvarez V, Torres J, Parra R. Secondary metabolites from Chamaedora tepejilote (Palmae) are active against Mycobacterium tuberculosis. Phytother Res 2005;19(4):320-2.

110. Gabay O, Sanchez C, Salvat C, Chevy F, Breton M, Nourissat G, et al. Stigmasterol: a phytosterol with potential anti-osteoarthritic properties. Osteoarthritis Cartilage 2010;18(1):106-16.

111. Saludes JP, Garson MJ, Franzblau SG, Aguinaldo AM. Antitubercular constituents from the hexane fraction of Morinda citrifolia Linn. (Rubiaceae). Phytother Res 2002;16(7):683-5.

112. Firdous S, Khan K, Zikr-Ur-Rahman S, Ali Z, Soomro $\mathrm{S}$, Ahmad VU, et al. Isolation of phytochemicals from Cordia rothii (Boraginaceae) and evaluation of their immunomodulatory properties. Rec Nat Prod 2014;8:51-5.

113. Ghannadian M, Akhavan A, Abdalla M, Ayatollahi AM, Mohammadi-kamalabadi M, Ghazanfari H. Triterpenes from Euphorbia spinidens with immunomodulatory activity. Res Pharm Sci 2013;8(3):205-10.

114. Gu JQ, Wang Y, Franzblau S, Montenegro G, Yang D, Timmermann BN. Antitubercular constituents of Valeriana laxiflora. Planta Med 2004;70:509-14. 